\title{
Application of the Curvature operator: Matrix Elements and properties of the new Hamiltonian Constraint operator in LQG
}

\section{Mehdi Assanioussi*}

Institute of Theoretical physics, University of Warsaw

E-mail: mehdi.assanioussiefuw.edu.pl

\begin{abstract}
This talk is a presentation of an ongoing work based on [1] of the same authors. In this work we study properties of the Lorentzian Hamiltonian constraint operator expressed using the curvature operator introduced in [1] and we evaluate its action.

References

[1] E. Alesci, M. Assanioussi and J. Lewandowski, A curvature operator for $L Q G$, Submitted for publication in Phys. Rev. D [arxiv: 1403.3190v2].
\end{abstract}

Frontiers of Fundamental Physics 14 - FFP14,

15-18 July 2014

Aix Marseille University (AMU) Saint-Charles Campus, Marseille

\footnotetext{
${ }^{*}$ Speaker.
} 